\title{
Tele-Protection Implementation Using IEEE 1588 Precision Time Protocol in Colombian High Power Substations
}

\author{
Hermes Eslava1, Luis Alejandro Rojas ${ }^{1}$, Danny Pineda ${ }^{2}$ \\ ${ }^{1}$ Universidad Distrital, Bogotá, Colombia \\ ${ }^{2}$ Openlink Sistemas DE Redes DE Datos S.A.S, Bogotá, Colombia \\ Email: hjeslavab@udistrital.edu.co
}

Received November 2014

\begin{abstract}
Optical fiber has been used as typical physical transmission medium in Colombian high power substations for effective communication between substations and control centers. However, suitable transmissions of tele-protection services continues to be a challenging area in the electricalsupply sector since link stability as well as various performance tests suggest that link recurrence requirements still exhibit limitations when using Metroethernet networks in this context. At present, improvements have been achieved via proprietary protocols such as Mirrored Bits, which matches the performance of a tele-protection link transmission under normal operation conditions. However, this imposes commercial limitations on the Colombian electrical-supply sector since proprietary protocols hinder fair competition among manufacturers. This is the rationale behind conducting trials with precision time protocol as it is defined in IEEE 1588 standard. The main purpose is to validate whether, under real operation conditions, it is possible to substitute such tele-protection links via Metroethernet networks, yet considering a standard protocol that allows incorporating various pieces of equipment from different manufacturers into the system as well as providing common benefits derived from using this type of communication networks. The present work provides technical results obtained after conducting various simulated tele-trigger tests, including stress tests, where results-stability validation occurs through network-saturation simulations. These saturation conditions are achieved by incorporating additional traffic, which involves several switching events over link paths and permits validating tele-protection-response timing when specifically using IEEE 1588 precision time protocol. Based on the aforementioned reasons as well as on the resulting benefits, a series of laboratory tests have been defined in order to evaluate delay and availability on tele-protection services when transported over IP networks.
\end{abstract}

\section{Keywords}

Metro Ethernet, IEEE 1588, IEC 61850, Tele-Protection, Effective Communications, Tele-Trigger Simulations 


\section{Introduction}

The present paper provides technical results obtained after conducting various simulated tele-trigger tests, including stress tests, where results-stability validation occurs through network-saturation simulations. These saturation conditions are achieved by incorporating additional traffic, which involves several switching events over link paths and permits validating tele-protection-response timing when specifically using protocol IEEE 1588. Based on the aforementioned reasons as well as on the resulting benefits, a series of laboratory tests have been defined in order to evaluate delay and availability on tele-protection services when transported over IP networks. This paper also provides detailed explanations on the following aspects: test setup, potential results, devices, and quality of Service schemes. The final part of the paper summarizes and discusses the most relevant results.

\section{Problem Formulation}

As multi-service complexity increases within connectivity-oriented networks (so called Metro Ethernet), such networks must support a wide range of services and applications that include large-scale traffic mechanisms in order to successfully provide time-sensitive services such as conventional telephony and VoIP. Likewise, requirements also become more demanding for the failure-avoidance equipment that protects the various system elements; preserving system stability. Ethernet architecture allows detecting errors, and although it is unable to make corrections, it does prevent damage to key devices within a network. In this context, tele-protection plays an important role in successfully disconnecting the affected parts of a system by means of quick signal transmissions.

The use of this type of networks has been implemented in order to promote features such as simplicity, compatibility, equity, stability, maintenance, and flexible addressing schemes over a multi-layer architecture; also including short delays, high speed and low cost [1]. This is the rationale behind the increased number of implementations of these networks, as indicated in a comparative study conducted by Cisco Systems, Inc. [2]. At this point it is worth asking what the connection is between Metro-Ethernet networks and tele-protection; can implementation of such networks have an impact on tele-protection systems? Tele-protection systems are based on tele-communication channels that provide deterministic signal-transmission delays together with constant bit rates (bandwidth) in time with no delay variations. Static multiplexing techniques, like those associated to Plesiochronous Digital Hierarchies (PDH) and Synchronous Digital Hierarchies (SDH), meet this requirement and so have been applied for decades by many companies when establishing their wide-area networks. Table 1 shows comparison between Ethernet and other technologies.

Thus, in various situations where Ethernet/IP-based networks are used for protection signaling, it is necessary to implement a solution that allows supervising the availability and quality of WAN communication channels (wide area network) and also send alert signals to the protection equipment in case reliable remote-terminalcommand transmissions were not guaranteed [3].

Table 1. Ethernet Compared to other alternatives.

\begin{tabular}{cccc}
\hline \multirow{2}{*}{ Technological Differences } & \multicolumn{2}{c}{ Alternatives } & ATM \\
\cline { 2 - 4 } Scalability & Ethernet & FR & $1.5 \mathrm{M}$ a $622 \mathrm{M}$ \\
QoS & $10 \mathrm{M} \mathrm{a} 10 \mathrm{G}$ & $56 \mathrm{~K} \mathrm{a} 45 \mathrm{M}$ & Yes \\
Service Flexibility & Supported & Limited & Low \\
Protocol Efficiency & High & Low & Low \\
IP-Optimized & High & Mid & No \\
Provisioning & Yes & No & Slow \\
CPE (Cost per Engagement) & Fast & Slow & $\$ \$ \$$ \\
Cost/Mb & $\$$ & $\$ \$$ & $\$ \$ \$$
\end{tabular}

${ }^{\mathrm{a}}$ Technological differences where Ethernet outperforms other alternatives. 


\section{Tele-Protection in Electric Substations}

At present, electrical energy companies focus on the potential risks of using non-deterministic Ethernet to transmit protection signaling at substations. This may cause failure in electrical supply systems, leading to voltage sags or service interruptions that have a negative impact on every-day-life activities and, to a worse extent, have serious financial consequences.

The main purpose of protection systems is to quickly isolate (take out of service) from the system any piece of high-power equipment that shows faulty behavior. Likewise these protection systems must limit the extent of damage caused to equipment and disconnect the faulty equipment as quickly as possible in order to keep stability and preserve the good condition of the whole power system. Since transient stability is closely related to the capabilities of the power system to maintain synchronization when undergoing massive perturbations, satisfactory behavior of protection systems is important in order to ensure stability.

In order to serve its purpose, a protection system must be capable of detecting variations that may occur around a given equilibrium point, reducing uncertainty and undefined states as much as possible. Such a system should also detect anomalies in specific components or pieces of power equipment as well as and limiting the time span of any anomaly (minimizing undesired delays). In conclusion, protection must contribute to the following desired features: 1) availability (\% of time estimated for equipment, or parts of the power system, to operate properly and be fully available), 2) reliability (probability that a piece of equipment, or part of the system, is fully operational with no failure events within a given time span) and 3) instability (ability to recover a previous operation state as a synchronous operation of generators after disturbances) [4].

Classification of different types of protection falls into three main groups. The first group corresponds to transmission-line protection, where the following devices are used: over-current breakers (either time-set or instant-response, directional, non-directional, or directional with communication capabilities), differential line breakers and distance breakers (with or without communication). The second group corresponds to distance breaker protection, which is normally installed in places where failure-isolation instantaneous response times are set. This second group allows larger instantaneous operation zones, greater sensitivity, easy adjustment, easy coordination, and offers particular advantages such as remaining unaffected by configuration changes in the power system. Lastly, the third group corresponds to power oscillation protection. This type of protection provides a balance between what is generated and what is consumed in terms of active and reactive power. Within an electrical power system, in stationary regime, any change in either the generated, the power demand, or in the electrical system itself, causes changes in the corresponding power transfer, which in turn oscillates until reaching a new equilibrium point between the generation system and the load.

Typical power system response to disturbances depends on configuration and also on the extent of perturbations. Depending on the type of disturbance as well as on the counteractions of protection equipment and control systems, electrical systems may remain stable and continue their normal operation around a new equilibrium point. This type of oscillation is referred to as stable-power oscillation. In case of system instability due to disturbances, a separation of rotor angles occurs in some generators, accompanied by power oscillations that lead to synchronization losses among generators (or among interconnected systems). This type of oscillation is called instable power oscillation [5].

\section{Laboratory Equipment and Application}

Considering the aforementioned conceptual framework as well as the operation of Metro-Ethernet networks, the structure of standard IEC61850 and IEEE 1588 precision time protocol and the benefits of applying tele-protection; the following section describes the equipment that constitutes a complete laboratory installed at the facilities of Openlink in Bogotá (Colombia). The main laboratory components are the following:

\subsection{CISCO ME 3400 Series Ethernet Access Switches}

Built for service providers, these multilayer customer-located switches allow you to deploy Ethernet-to-thehome (ETTH) “triple play” services and Ethernet-to-the-business (ETTB) VPN services. Based on today's most widely deployed access switches, the Cisco Catalyst 2950 and 3550 Series, the Cisco ME 3400 Series provides service-provider-friendly hardware and mission-specific software [6] a single ETTH and ETTB access solution to help reduce total ownership costs and operating expenses, compact form factors for ease of deployment, only 
front connectors to simplify access (except for ME-3400G-2CS-A), NEBS Level 3 and ETSI certification (ME3400G-2CS-A is only NEBS3 certified), Metro Ethernet Forum (MEF) 9 and 14 certification and support for multiple software images. The switches feature comprehensive security capabilities network based security to protect the network from unauthorized traffic, switch security to help maintain continuous switch operation and subscriber security to shield subscribers from other malicious users.

\subsection{CISCO 2500 Connected Grid Switches}

The Cisco 2500 Series Connected Grid Switch is a rugged switch optimized for use in transmission and distribution (T\&D) power substations. The Cisco 2520 Connected Grid Switch (CGS 2520) is designed for substation networks to endure harsh environments, common in transmission and distribution substations. The CGS 2520 uses Cisco IOS Software, the operating system that powers millions of Cisco switches worldwide [7]. Below primary features:

- Rugged industrial design and substation compliance: IEC-61850-3 and IEEE 1613 for utility substation environments

- No fans, no moving parts for maximum reliability

- Tools for easy deployment, management, and replacement

- Extensive instrumentation and remote diagnostic capabilities

- Advanced Cisco IOS Software features such as 802.1x, Layer 2-Layer 4 access control lists, port security, and more

- Advanced QoS capabilities to support mission-critical substation applications such as supervisory control and data acquisition (SCADA) and IEC 61850 Generic Object-Oriented Substation Events (GOOSE) messaging

- Comprehensive network security features based on open standards

- Field-replaceable components such as power supplies and Small Form-Factor Pluggables (SFPs)

- Intuitive graphical user interface (GUI) using Cisco Configuration Professional

In addition, the 2520 Connected Grid Switch has REP and Flexlink, which provide fast re-convergence in ring and hub-and-spoke topologies [8], and smart-port templates provide one-touch global and port-level macros to simplify switch deployments. There are two models for added flexibility first 24-port 10/100TX with dual-purpose gigabit Ethernet uplinks and 24 ports of mixed fiber and copper, including 16 fast Ethernet fiber SFP ports and 810/100 Power over Ethernet (PoE) ports with dual-purpose gigabit Ethernet uplinks.

In addition to these two essential pieces of equipment Figure 1, within the laboratory design we also included general components such as routers, remote terminal units (to capture information whenever event/second directive triggers occurred), multiplexers, oscilloscopes (with terminals intended to capture delay-associated data) and a network manager (to supervise the acquired statistical data). The 24-Volts-per-time-unit input (one second in this case), allows $200 \mathrm{~ms}$, providing the necessary interruption time span required by the system [9]. The voltage-measurement map associated to source generation for tele-protection equipment electrical connections is shown in Figure 2:

\section{Results}

Regarding overall results, it is worth mentioning that:

- In virtually all cases (99.96\%), maximum end-to-end delay remained below 9 ms (at tele-protection equipment terminals). Moreover, $99.99 \%$ of the times delay did not exceed $25 \mathrm{~ms}$.

- During the longest tests (22.7 days capturing data and using congestion-drive devices), significant availability (99.987\%) of trigger events was achieved (100\% availability is not achievable due to unavailability periods, non-defined for the model within working hours).

- Results are based on a very large number of samples. All the cases analyzed herein include over 2 million samples.

\section{Conclusions}

In conclusion, the most relevant aspect of an electrical-energy substation lies in the protection of electrical lines. 


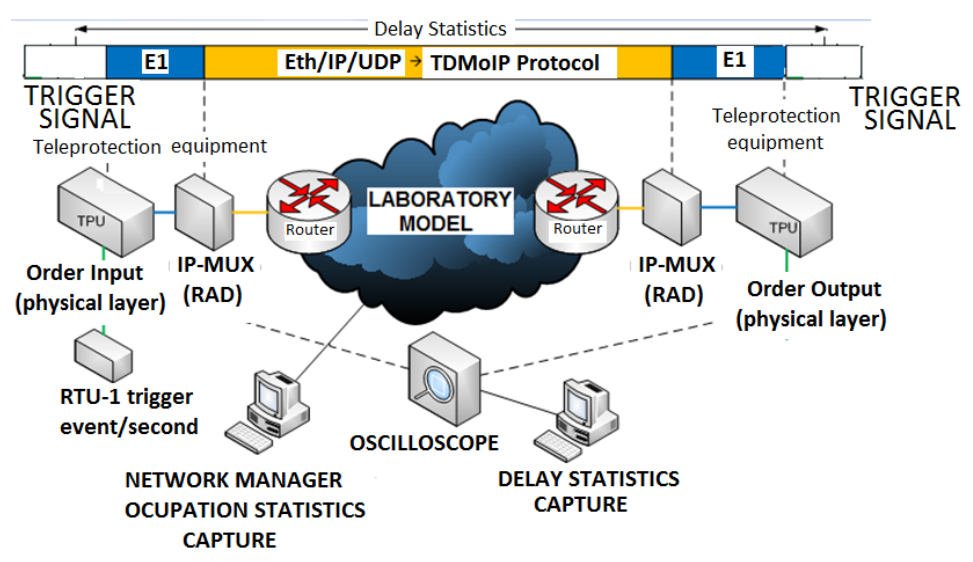

Figure 1. IP teleproteccion test model-laboratory (installed equipment).

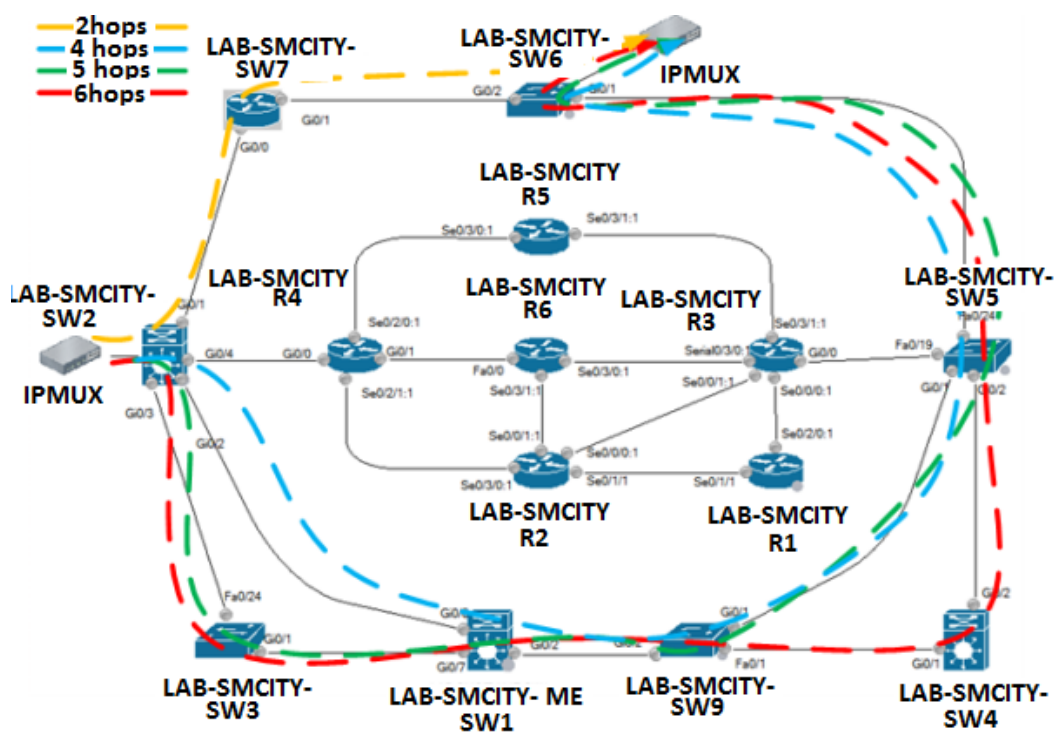

Figure 2. IP tele-protection test model-measurement and source-voltage-generating electrical connections to the tele-protection equipment.

To successfully achieve this purpose, nowadays, there is sophisticated equipment based on reliable sensors that are capable of measuring hundreds of line parameters on a regular basis, supported by implementation of standard IEC61850 and IEEE 1588.

\section{Acknowledgements}

The present study was conducted at Openlink laboratories in Bogotá (Colombia). At present, advances are being developed regarding the identification of configurations and patterns that comply with international regulations on the deployment of grids that cover this type of services. Based on the present is study, electrical-energy distribution companies have begun adopting this technology as well as deploying Metro-Ethernet networks using robust, highly reliable optical-fiber links. The authors would like to thank Openlink for the permanent support throughout this research project, particularly in its various stages, namely the technological survey, the search for target applications and the process of acquiring experience in the field of Ethernet-network protection complying with international standards.

\section{References}

[1] Martin, A. and Francisco, G. (2013) Temas específicos de comunicaciones II: Ethernet/IP. 
http://www.monografias.com/trabajos-pdf2/ethernet-ip/ethernet-ip.pdf

[2] Comino, R. and Stittmatter, M. (2011) Protección avanzada de redes eléctricas: La próxima generación de soluciones de teleprotección. ABB revista, 2-8.

[3] I. Cisco Systems (2005) Servicios y Soluciones Metro Ethernet. Cisco Systems, Inc.

[4] Calero, R. (2007) Laboratorio de comunicaciones: Redes Metro Ethernet. FIUBA, Buenos Aires.

[5] UNAM (2011) Consideraciones sobre el Protocolo IEC-61850. UNAM, Ciudad de Mexico.

[6] IEC61850 (2008) Communication Networks and Systems for Power Utility Automation. International Electrotechnical Commission, IEC 61850, 2, 57/942/CDV.

[7] Fing (2007) Estabilidad de Sistemas Eléctricos de Potencia. In: Capítulo 5: Estabilidad Transitoria, Protecciones, Montevideo, Universidad de la República, Instituto de Ingeniería Eléctrica, 2-31.

[8] Sena, C. (2010) Tesis Oscilación de potencia en los sistemas eléctricos: protección y control, Montevideo: Universidad de la República, Maestría en Ingeniería Eléctrica, 6-19.

[9] Villalón, E.P. (2008) Diseño y Optimización de una Arquitectura IEC61850. ESCUELA TÉCNICA SUPERIOR DE INGENIERÍA (ICAI), Madrid, Junio. 